\title{
Differenzierung von Angststörungen im Elternurteil: Untersuchung anhand des Fragebogens für Angst- und Zwangsstörungen (FBB-ANZ)
}

\author{
Andreas M. Mühlherr, Afsheen Yousaf und Christine M. Freitag \\ Klinik für Psychiatrie, Psychosomatik und Psychotherapie des Kindes- und Jugendalters, Klinikum der Goethe-Universität, \\ Frankfurt am Main
}

\begin{abstract}
Zusammenfassung: Im Rahmen der Abklärung des Verdachts einer Angststörung kommen in der klinischen Praxis regelmäßig Screeningfragebögen für Eltern zum Einsatz, die teilweise auch unterschied liche Angststörungen erfassen. Im klinischen Kontext ist die valide Abgrenzung von Angststörungen zu anderen psychischen Störung sowie die differenzialdiagnostische Abklärung spezifischer Angststörungen relevant. Das Ziel der vorliegenden Studie ist die Untersuchung der Validität eines Screenings durch den Fremdbeurteilungsbogen für Angst- und Zwangsstörungen (FBB-ANZ) hinsichtlich unterschiedlicher Angststörungen. Überprüft wurde die Diskriminationsfähigkeit von (1) Angststörungen und anderen kinder- und jugendpsychiatrischen Störungen und (2) verschiedenen Angststörungen mittels ROC-Analysen (Receiver Operating Characteristics). Der FBB-ANZ wurde von 972 Eltern von 4;00-11;11-jährigen Kindern und 12;00-17;11-jährigen Jugendlichen mit Angststörungen oder depressiven Episoden oder externalisierenden Störungen ausgefüllt. Die Diskriminationsfähigkeit von Angststörungen und externalisierenden Störungen bei Kindern (AUC [Area Under the Curve] = .72) und Jugendlichen (AUC = .76) sowie von Angststörungen und depressiven Episoden im Kindesalter (AUC = .77) war moderat. Eine gute Unterscheidung verschiedener Angststörungen bei Angstpatient_innen war nur hinsichtlich der emotionalen Störung mit Trennungsangst bei Kindern (AUC $=.84)$ und Jugendlichen (AUC = .87) gegeben. Die Ergebnisse deuten auf einen eingeschränkten diagnostischen Nutzen des Screeningurteils der Eltern zur Unterscheidung verschiedener Angststörungen im Kindes- und Jugendalter hin. Mögliche Erklärungsansätze für die vorliegenden Ergebnisse werden kritisch diskutiert.
\end{abstract}

Schlüsselwörter: Angststörungen, Kindes- und Jugendalter, Screeningfragebogen, Elternurteil, ROC-Analysen

Discrimination of anxiety disorders by parent ratings: A study based on the Questionnaire for Anxiety and Obsessive-Compulsive Disorders (FBB-ANZ)

Abstract: Parent ratings are often used for screening during the diagnostic evaluation of anxiety disorders. Clinically, it is important to correctly differentiate between anxiety and other psychiatric disorders and to distinguish specific anxiety disorders. The present study examined the validity of the screening results obtained by the Parent Questionnaire for Anxiety and Obsessive-Compulsive Disorders (FBB-ANZ). We examined whether the FBB-ANZ discriminated (1) anxiety and other psychiatric disorders and (2) specific anxiety disorders in children and adolescents using ROC analyses. 972 parents of 4;00-11;11-year-old children and 12;00-17;11-year-old adolescents with anxiety disorders, depressive episodes, or externalizing disorders completed the FBB-ANZ. Discrimination of anxiety disorders and externalizing disorders in children $(A \cup C=.72)$ and adolescents $(A \cup C=.76)$ as well as depressive episodes in children (AUC $=.77)$ was moderate. Good discrimination of different anxiety disorders was found only for separation anxiety in children (AUC $=.84$ ) and adolescents $(A \cup C=.87)$. The results indicate the limited diagnostic benefit of parent ratings for discriminating different anxiety disorders in children and adolescents. Potential explanations for the results are critically discussed.

Keywords: anxiety disorders, children and adolescents, screening questionnaire, parent rating, ROC analyses

Angststörungen gehören zu den häufigsten psychiatrischen Störungen des Kindes- und Jugendalters. In einer populationsbasierten Erhebung des Robert-Koch-Instituts aus Deutschland lagen die geschätzten Prävalenzen für alle Angststörungen innerhalb der Gruppe der 7- bis 11-Jährigen bei $6.3 \%$ und in der Gruppe der 11- bis 17-Jährigen bei $4.0 \%$
(Ravens-Sieberer et al., 2008). Zwischen den verschiedenen Angststörungen zeigen sich Unterschiede in den Häufigkeiten und dem Ersterkrankungsalter (Barkmann \& Schulte-Markwort, 2007; Ihle \& Esser, 2002). Die emotionale Störung mit Trennungsangst, spezifische Phobien und soziale Phobien haben ihren Beginn meist im Kindesalter 
(Beesdo, Knappe \& Pine, 2009). Populationsbasierte Untersuchungen berichten 6-Monats-Prävalenzen von Trennungsänsten nach DSM-III-R von $4.9 \%$ bei 6- bis 8-jährigen Kindern und von $1.3 \%$ bei 12 - bis 14 -jährigen Jugendlichen (Breton et al., 1999). Bei sozialen Phobien nach DSM-IV liegt die Lebenszeitprävalenz 14- bis 17-jähriger Jugendlicher bei $4 \%$ (Wittchen, Stein \& Kessler, 1999). Die Häufigkeiten von spezifischen Phobien liegen zwischen 2.6 und 9.1\% (Ollendick, King \& Muris, 2002). Die Agoraphobie und die generalisierte Angststörung (GAS) haben ihren Beginn meist im Jugendalter. Die Lebenszeitprävalenz der DSM-IV-Diagnose Agoraphobie liegt bei 14bis 17-jährigen Jugendlichen bei $2.8 \%$ (Beesdo et al., 2009). Bei der generalisierten Angststörung liegt die 3-Monats-Prävalenz im Kindesalter bei $1.8 \%$ und im Jugendalter bei $4.0 \%$ (Beesdo et al., 2009).

Zwischen den verschiedenen Angststörungen werden zudem hohe Komorbiditätsraten berichtet (Last, Strauss \& Francis, 1987). In einer Untersuchung an 7- bis 17-jährigen Patient innen mit mindestens einer der DSM-IV-TR-Diagnosen soziale Phobie, Trennungsangst oder generalisierte Angststörung erfüllten $41.7 \%$ der Kinder und $20.6 \%$ der Jugendlichen die Kriterien aller drei Störungsbilder (Kendall et al., 2010). Neben Angsterkrankungen gehören depressive Störungen und die Aufmerksamkeitsdefizit-/Hyperaktivitätsstörung (ADHS) zu den häufigsten kinder-und jugendpsychiatrischen Erkrankungen (Ihle \& Esser, 2002). Bei ADHS werden im Kindes- und Jugendalter Prävalenzen bis $7 \%$, bei depressiven Erkrankungen bis $8 \%$ berichtet (Flemming \& Offord, 1990; Sayal, Prasad, Daley, Ford \& Coghill, 2018). Depressive Störungen und ADHS sind zusätzlich die häufigsten nicht angstbezogenen komorbiden Störungen von Angsterkrankungen (Essau, Conradt \& Petermann, 2000; Kendall et al., 2010). In Untersuchungen von Patientenpopulationen lag bei 8 bis 13 Jahre alten Kindern mit Angststörungen in 17\% der Fälle komorbides ADHS vor (Verduin \& Kendall, 2003). Bei 12- bis 17-jährigen Jugendlichen mit Angststörungen erfüllten $30 \%$ die Kriterien depressiver Störungen (Essau et al., 2000), bei 7- bis 13-jährigen Kindern mit sozialen Phobien wurden in $6 \%$ der Fälle komorbide depressive Störungen und in $10 \%$ der Fälle komorbides ADHS berichtet (Beidel, Turner \& Morris, 1999). Bei 13- bis 16-jährigen Jugendlichen mit sozialen Phobien zeigten sich in $8 \%$ der Fälle depressive Störungen und in $4.8 \%$ der Fälle ADHS (Beidel et al., 2007).

Die hohen Prävalenzraten von Angststörungen und das häufige komorbide Auftreten verschiedener Angststörungen stellen hohe Anforderungen an die differenzialdiagnostische Urteilsbildung. Weiter erschweren Überlappungen im klinischen Phänotyp von Angststörungen mit anderen, häufig komorbiden internalisierenden und externalisierenden Störungen die differenzialdiagnostische
Einordnung. Beispielsweise sind Selbstwertprobleme sowohl mit Angststörungen als auch mit depressiven Erkrankungen assoziiert (Iancu, Bodner \& Ben-Zion, 2015; Orth, Robins \& Roberts, 2008; Sowislo \& Orth, 2013). Negative Affektivität ist Teil depressiver Störungen und von Angsterkrankungen (Cummings, Caporino \& Kendall, 2014). Zur Unterstützung des diagnostischen Prozesses hat sich der Einsatz standardisierter Screeningfragebögen als effiziente, kostengünstige und zeitsparende Methode etabliert. Um die Ergebnisse von Screeningfragebögen im diagnostischen Prozess korrekt interpretieren zu können, sind Untersuchungen zur externen, diskriminanten Validität notwendig. Im klinischen Kontext ist es insbesondere von Interesse, eine hohe Validität hinsichtlich der Differenzialdiagnostik zu anderen psychischen Störungen sowie hinsichtlich des Vorliegens einer spezifischen Angststörung zu erreichen.

Ziel der vorliegenden Studie ist deshalb die Überprüfung der externen Validität des elterlichen Screeningergebnisses anhand des Fremdbeurteilungsbogens für Angst- und Zwangsstörungen (FBB-ANZ) des DiagnostikSystems für psychische Störungen nach ICD-10 und DSM-IV für Kinder und Jugendliche - II (DISYPS-II; Döpfner, GörtzDorten \& Lehmkuhl, 2008), ein in der ambulanten und stationären Versorgung häufig eingesetztes Instrument. Als Besonderheit der kinder- und jugendpsychiatrischen Diagnostik ist die Beurteilung von Symptomen durch die Eltern eine wichtige Informationsquelle und zeigt große Übereinstimmung mit dem klinischen Urteil (Grills \& Ollendick, 2003). Da sich Angstsymptome und die Prävalenzen spezifischer Angsterkrankungen zwischen Kindern und Jugendlichen unterscheiden (Kashani \& Orvaschel, 1990; Waite \& Creswell, 2014), erfolgt die Untersuchung in zwei Altersklassen (Kindesalter 4;00-11;11 Jahre und Jugendalter 12;00-17;11 Jahre).

Zur Überprüfung der Gültigkeit der Ergebnisse in der vorliegenden Stichprobe wurden zunächst die Faktorenstruktur und die psychometrischen Eigenschaften des FBB-ANZ repliziert. Zur Bestimmung der externen Validität des FBB-ANZ hinsichtlich der Abgrenzung zu anderen psychischen Störungen im Kindes- und Jugendalter wurden zudem die Summenwerte des Fragebogens zwischen Patient_innen mit mindestens einer Angststörung und Patient_innen ohne Angststörungen, aber mit depressiven Erkrankungen oder externalisierenden Störungsbilder verglichen. Weiter wurde die Güte der Klassifikation des FBB-ANZ bei Angstpatient_innen im Vergleich zu Patient innen mit depressiven Erkrankungen oder externalisierenden Störungen, jeweils ohne komorbide Angststörungen, untersucht. Zusätzlich wurde untersucht, wie gut der Elternfragebogen Kinder und Jugendliche mit verschiedenen Angststörungen korrekt klassifiziert. 


\section{Methoden}

\section{Stichprobe mit Ein- und Ausschlusskriterien}

Eingeschlossen wurden 972 Kinder und Jugendliche zwischen 4;00-17;11 Jahren, die aufgrund einer psychiatrischen Erkrankung zwischen 2009 und 2018 in ambulanter oder (teil)stationärer Behandlung in der Klinik für Psychiatrie, Psychosomatik und Psychotherapie des Kindes- und Jugendalters des Universitätsklinikums der Goethe-Universität Frankfurt Main waren und deren Eltern der Datenauswertung zustimmten. Die pseudonymisierte Datenanalyse von klinisch erhobenen Daten anhand unterschiedlicher Fragestellungen wurde durch die Ethikkommission des Fachbereichs Medizin der Goethe-Universität Frankfurt bewilligt. Der verwendete Screeningfragebogen lag für alle Patient_innen mit weniger als $10 \%$ fehlende Werte der diagnoserelevanten Items 1 bis 33 vor. Die Patient_innen wurden von erfahrenen (Fach-) Ärzt_innen für Kinder- und Jugendpsychiatrie und -psychotherapie und/ oder (approbierten) Kinder- und Jugendlichenpsychotherapeut_innen gemäß ICD-10 (International Statistical Classification of Diseases and Related Health Problems, 1992) diagnostiziert. Patient_innen mit Angststörungen, Patient_innen mit depressiven Störungen und Patient_innen mit externalisierenden Störungen wurden verglichen. Einschlusskriterium in die Gruppe der Angststörungen war das Vorliegen mindestens einer der folgenden Diagnosen: F93.0 Emotionale Störung des Kindesalters mit Trennungsangst, F41.1 Generalisierte Angststörung, F40.1 Soziale Phobien/F93.2 Störung mit sozialer Ängstlichkeit, F40.2 Spezifische Phobien/F93.1 Phobische Störung des Kindesalters, F40.0 Agoraphobie mit/ohne Panikstörung oder F41.0 Panikstörung. Alle komorbiden Störungen waren zugelassen. In die Gruppe der Patient_innen mit depressiven Episoden wurden die Diagnosen einer depressiven Episode oder rezidivierenden depressiven Störung (F32.0/1/2; F33.0/1/2) eingeschlossen. Komorbide Angstoder externalisierende Störungen waren ausgeschlossen. Eingeschlossen in die Gruppe der externalisierenden Störungen wurden die Diagnosen F90.- Hyperkinetische Störungen, F91.- Störung des Sozialverhaltens, F92.- Kombinierte Störung des Sozialverhaltens und der Emotionen und F98.8 Aufmerksamkeitsstörung ohne Hyperaktivität. Angststörungen oder depressive Störungsbilder waren ausgeschlossen. In der Gruppe der Angststörungen wurden zusätzlich, analog zu den Angstskalen des verwendeten Screeningfragebogens, vier Untergruppen gebildet: Patient_innen mit F93.0 Emotionale Störung des Kindesalters mit Trennungsangst, mit F41.1 Generalisierte Angststörung, mit F40.1 Soziale Phobien/F93.2 Störung mit sozialer Ängstlichkeit oder mit F40.2 Spezifische Phobien/F93.1 Phobische Störung des Kindesalters.

\section{Erhebungsinstrumente}

\section{Fremdbeurteilungsbogen für Angst- und Zwangsstörungen (FBB-ANZ)}

Der FBB-ANZ (Döpfer et al., 2008) bildet die Diagnosekriterien nach ICD-10 und DSM-IV ab. Er umfasst 45 Items und erfasst Symptome einer emotionalen Störung mit Trennungsangst, einer generalisierten Angststörung, spezifischen Phobien, sozialen Angststörungen, Agoraphobie und von Zwangsstörungen im Elternurteil. Die Antworten auf den diagnoserelevanten Items erfolgen auf einer vierstufigen Skala $(0=$ trifft nicht $z u, 1=$ trifft teilweise $z u, 2=$ trifft sehr $z \mathrm{u}, 3=$ trifft stark $z u)$. Die Mittelwerte der Items 1 bis 10 bilden die Skala Trennungsangst, 11 bis 17 die Skala Generalisierte Angst, 18 bis 24 die Skala Soziale Phobie, 25 bis 31 die Skala Spezifische Phobie und 1 bis 33 die Gesamtskala Angst und Zwang. Die Zwangsitems 32 und 33 des Fragebogens wurden für die vorligenende Untersuchung nur als Teil der Gesamtskala Angst und Zwang, nicht aber als eigene Skala untersucht. Es liegen alters- und geschlechtsspezifische Normen für 4- bis 6-jährige, 7- bis 10-jährige, 11- bis 13-jährige und 14- bis 17-jährige Mädchen und Jungen vor.

\section{Child Behavior Checklist (CBCL)}

Die CBCL (Achenbach, 1991; Arbeitsgruppe Deutsche Child Behavior Checklist, 1998) ist ein standardisierter Fragebogen zur Erfassung von Verhaltensauffälligkeiten, somatischen Beschwerden und emotionalen Problemen bei 4- bis 18-jährigen Kindern und Jugendlichen im Elternurteil. Die CBCL lag für 904 Patient_innen vor. Neben der klinischen Diagnose wurden als weitere externe Validitätskriterien die Subskalen Ängstlich/depressiv, Sozialer Rückzug und Internalisierende Störungen untersucht.

\section{Intelligenzdiagnostik}

Von 652 Patient_innen liegen Ergebnisse einer Intelligenzdiagnostik mit folgenden Verfahren vor (Fallzahlen in Klammer): Culture Fair Test - Grundintelligenz Skala 1 (CFT-1-R; Weiß \& Osterland, 2012) (2), Culture Fair Test - Grundintelligenz Skala 2 (CFT 20-R; Weiß, 2006) (38), Hannover-Wechsler-Intelligenztest für das Vorschulalter-III (HAWIVA-III; Ricken, Fritz, Schuck \& Preuß, 2007) (20), Wechsler Intelligenztest für Kindergartenkinder und Vorschulkinder-III (WPPSI-III; Petermann, 2009) (4), Hamburg-Wechsler-Intelligenztest für Kinder-III (HAWIK-III; Tewes, Rossmann \& Schallberger, 1999) (4), Wechsler Intelligenz-Test für Kinder-IV (WISC-IV; Petermann \& Petermann, 2011) (506), Wechsler Intelligenz-Test für Erwachsene-III (WAIS-III; Wechsler, 1997) (1), Wechsler Intelligenztest für Erwachsene (WIE; Aster, Neubauer \& Horn, 2006) (21) und Wechsler Intelligenztest für Erwachsene-IV (WAIS-IV; Petermann, 2012) (16). 


\section{Statistische Auswertung}

\section{Itemanalyse und interne Konsistenz}

Die Berechnungen erfolgten mit IBM SPSS Statistics 24 . Die Trennschärfen wurden zwischen allen Items und der Gesamtskala sowie den Subskalenitems und dem Gesamtwert der störungsspezifischen Subskalen berechnet (Bortz \& Döring, 2006). Die Itemschwierigkeiten wurden mit dem Schwierigkeitsindex für intervallskalierte Daten (Wertebereich 0 bis 100) bestimmt (Moosbrugger \& Kelava, 2012). Die Berechnung der internen Konsistenzen für die Gesamtskala sowie für die vier Subskalen des Fragebogens erfolgte über Cronbachs Alpha.

\section{Konfirmatorische Faktorenanalyse}

Für die konfirmatorische Faktorenanalyse (CFA) mit $N=972$ wurden fehlende Werte durch Multiple Imputation (MI) ersetzt. Die MI ist als robuste Methode zum Umgang mit fehlenden Werten auch bei verletzter Normalverteilungsannahme und intervallskalierten Daten anwendbar (Leite \& Beretvas, 2010). Die MI mit R-3.4.4 mice (van Buuren \& Groothuis-Oudshoorn, 2011) basierte auf den Daten des FBB-ANZ, Alter, Geschlecht, IQ und den Diagnosegruppen. Es wurden fünf Datensätze auf Basis von fünf Schätzungen durch predictive mean matching (pmm) erzeugt und zusammengefasst (Rubin, 1987; Vink, Lazendic \& van Buuren, 2015). Die CFA wurde mit R-3.4.4 lavaan (Rossel, 2012) durchgeführt. Die Modellüberprüfung erfolgte mit der Diagonally Weighted Least Squares (DWLS)-Schätzmethode (Flora \& Curran, 2004; Li, 2016). Berichtet werden die Ergebnisse eines $\chi^{2}$-Tests, die relativen Gütemaße Comparative Fit Index (CFI), Tucker-Lewis Index (TLI) und Normed Fit Index (NFI) und die absoluten Gütemaße Root Mean Square Error of Approximation (RMSEA) und Standardized Root Mean Square Residual (SRMR). Ein nicht signifikantes Ergebnis des $\chi^{2}$-Tests deutet auf eine Übereinstimmung des Messmodells mit den Daten hin. Ein CFI $\geq .90$, ein TLI $\geq .90$ und ein NFI zwischen .90 und .95 deuten auf eine akzeptable, ein CFI $\geq .95$, ein TLI $\geq .95$ und ein NFI $>.95$ deuten auf eine gute Übereinstimmung zwischen Modell und Daten hin. Ein RMSEAWert $<.08$ und ein SRMR-Wert $<.08$ deuten auf eine akzeptable bis gute Übereinstimmung zwischen Daten und Messmodell hin (Brown \& Cudeck, 2016; Hooper, Coughlan \& Mullen, 2008; Hu \& Bentler, 1999). Im Sinne einer Sensitivitätsanalyse wurde die Berechnung im nicht imputierten Datensatz wiederholt.

\section{Validität}

Korrelationsuntersuchungen mit CBCL-T-Werten erfolgten mit Pearsons-Korrelationskoeffizienten. Gruppenunterschiede in den (Sub-)Skalenwerten wurden aufgrund nicht erfüllter Voraussetzungen für parametrisches Tes- ten durch nonparametrischen Mittelwertvergleich (Kruskal-Wallis-Test; Mann-Whitney-U-Test) berechnet. Die Diskriminationsfähigkeit des FBB-ANZ wurde durch Receiver Operating Characteristics-Analysen (ROC-Analysen) in IBM SPSS Statistics 24 bestimmt. ROC-Analysen ermöglichen die Untersuchung der Klassifikationsgüte eines Fragebogens anhand des Verhältnisses von Richtigund Falsch-positiven Ergebnissen beim Vergleich zweier Diagnosegruppen über die Area Under the Curve (AUC). Zur Differenzierung von Angststörungen und depressiven Episoden oder externalisierenden Störungen wurde der Summenwert der Gesamtskala verwendet. Zur Differenzierung verschiedener Angststörungen wurde der Summenwert der entsprechenden Subskala herangezogen. Es folgte die Bestimmung der optimalen (Sub-)Skalenwerte (1) bei einer Sensitivität von ca. 90\% sowie (2) anhand des Youden-Index (Maximierung von Sensitivität und Spezifität).

\section{Resultate}

\section{Deskriptive Statistik}

Die deskriptiven Statistiken der Stichprobe sind Tabelle 1 zu entnehmen. In der Altersgruppe der 4;00-11;11-Jährigen unterschieden sich Patient_innen mit Angststörungen von Patient_innen mit depressiven Episoden hinsichtlich des Alters $(t[169]=1.98, p=.05)$, jedoch nicht hinsichtlich IQ $(t[482]=0.71, p=.48)$ oder Geschlechterverteilung $\left(\chi^{2}=0.19, p=.66\right)$. Zwischen Patient_innen mit Angststörungen oder externalisierenden Störungen gab es keine Unterschiede hinsichtlich des Alters $(t[343]=1.45, p=.15)$ oder IQ $(t[254]=0.12, p=.91)$, jedoch beim Geschlechterverhältnis $\left(\chi^{2}=22.43, p<.00\right)$. In der Altersgruppe der 12;00-17;11-Jährigen unterschieden sich Patient_innen mit Angststörungen und Patient_innen mit depressiven Episoden nicht hinsichtlich Alter $(t[488]=1.04, p=.31)$, IQ $(t[303]=0.38, p=.70)$ oder Geschlechterverteilung $\left(\chi^{2}=0.80, p<.37\right)$. Patient_innen mit externalisierenden Störungen waren jünger $(t[523]=7.39, p<.00)$, zeigten einen niedrigeren IQ $(t[342]=0.94, p<.00)$ und waren häufiger männlich $\left(\chi^{2}=42.20, p<.00\right)$ als die Patient_innen mit Angststörungen.

\section{Konfirmatorische Faktorenanalyse}

Die Beurteilung der Anpassungsgüte des Modells anhand des $\chi^{2}$-Tests auf Unabhängigkeit deutete auf eine fehlende Übereinstimmung des vierfaktoriellen Messmodells und den Daten hin $\left(\chi^{2}[489]=1937.19, p<.00\right)$. Die deskripti- 
Tabelle 1. Stichprobenbeschreibung

\begin{tabular}{|c|c|c|c|c|c|c|}
\hline & \multicolumn{2}{|c|}{ Alter } & \multicolumn{2}{|c|}{ Geschlecht } & \multicolumn{2}{|l|}{ IQ } \\
\hline & $\mathrm{n}$ & M (SD) & $\mathrm{n}$ & $(\% w)^{a}$ & $\mathrm{n}$ & $M(S D)$ \\
\hline \multicolumn{7}{|l|}{ 4;00-11;11 Jahre } \\
\hline Angststörungen & 163 & $8.84(2.13)$ & 163 & 45.4 & 118 & $96.03(16.89)$ \\
\hline - Trennungsangst & 39 & $9.25(1.92)$ & 39 & 30.8 & 28 & $98.18(16.17)$ \\
\hline - Soziale Phobien & 88 & $8.27(2.19)$ & 88 & 56.8 & 66 & $96.17(17.93)$ \\
\hline - Spezifische Phobien & 38 & $9.37(2.01)$ & 38 & 39.5 & 28 & $93.32(18.31)$ \\
\hline - Generalisierte Angststörung & 8 & $9.64(2.09)$ & 8 & 37.5 & 3 & $87.67(4.93)$ \\
\hline Depressive Episoden & 8 & $10.33(2.37)$ & 8 & 37.5 & 2 & $104.5(7.78)$ \\
\hline Externalisierende Störungen & 183 & $8.49(1.92)$ & 183 & 21.9 & 139 & $96.22(14.79)$ \\
\hline \multicolumn{7}{|l|}{ 12;00-17;11 Jahre } \\
\hline Angststörungen & 412 & $15.26(1.57)$ & 412 & 62.6 & 257 & $102.34(14.92)$ \\
\hline - Trennungsangst & 20 & $13.2(.98)$ & 20 & 65.0 & 12 & $104.48(12.97)$ \\
\hline - Soziale Phobien & 285 & $15.24(1.56)$ & 285 & 64.9 & 170 & $102.55(15.44)$ \\
\hline - Spezifische Phobien & 80 & $15.35(1.55)$ & 80 & 57.5 & 55 & $99.93(14.61)$ \\
\hline - Generalisierte Angststörung & 40 & $15.39(1.58)$ & 40 & 67.5 & 26 & $103.92(10.06)$ \\
\hline Depressive Episoden & 79 & $15.45(1.51)$ & 79 & 68.4 & 49 & $101.84(13.88)$ \\
\hline Externalisierende Störungen & 112 & $14.02(1.60)$ & 112 & 27.7 & 86 & $94.90(16.65)$ \\
\hline
\end{tabular}

Anmerkungen: Stichprobengröße ( $n$ ), Mittelwert (M) und Standardabweichung (SD).

a Prozentualer Anteil des biologisch weiblichen Geschlechts in den jeweiligen Gruppen.

ven Gütemaße zur Modellbeurteilung deuteten hingegen auf eine gute (SRMR $=.00$ ) bis akzeptable (RMSEA = .06; $\mathrm{CFI}=.93 ; \mathrm{TLI}=.92 ; \mathrm{NFI}=.91)$ Anpassungsgüte hin. Die Fit-Indizes im imputierten und nicht imputierten Datensatz zeigten hierbei keine Unterschiede (siehe elektronisches Supplement [ESM] 1). Aufgrund der Eigenschaft des $\chi^{2}$-Tests, bei steigender Stichprobengröße ein signifikantes Ergebnis zu liefern (Schermelleh-Engel, Moosbrugger \& Müller, 2003), wurde die Faktorenstruktur des Fragebogens als bestätigt angesehen und im Rahmen der Validitätsuntersuchung verwendet.

\section{Itemanalyse und interne Konsistenz}

Die Trennschärfen aller Items auf Gesamtskalenniveau lagen zwischen .26 und .66. Für die Subskala Trennungsangst lagen die Trennschärfen der einzelnen Items zwischen .44 und .70, für die Subskala Generalisierte Angststörung zwischen .40 und .67, für die Subskala Soziale Phobien zwischen .51 und .82 und für die Subskala Spezifische Phobien zwischen .32 und .55. Die Itemschwierigkeiten lagen zwischen 7 und 46. Die interne Konsistenz
(Cronbachs Alpha) der Gesamtskala lag bei .91 und für die Subskalen zwischen .71 und .89 (siehe ESM 2).

\section{Übereinstimmungsvalidität}

Die Gesamtskala Angst und Zwang des FBB-ANZ zeigte mittlere Pearson-Korrelationen mit den CBCL-T-Skalen Ängstlich/depressiv $(r=.57)$, Sozialer Rückzug $(r=.47)$, und Internalisierende Probleme $(r=.60)$.

\section{Gruppenvergleich der (Sub-)Skalenwerte und ROC-Analysen}

Die Mittelwerte, Standardabweichungen und Teststatistik der Gesamtskala Angst und Zwang und der Subskalen sind, getrennt nach Alters- und Patientengruppen, Tabelle $2 \mathrm{zu}$ entnehmen. Angstpatient_innen wiesen höhere Werte auf der Gesamtskala auf als Patient_innen ohne Angststörungen. Bei 4;00-11;11-jährigen Kindern unterschied der FBB-ANZ moderat zwischen Angststörungen und depressiven Episoden $(\mathrm{AUC}=.77)$ und zwischen 
Angststörungen und externalisierenden Störungen (AUC = .72). Bei 12;00-17;11-jährigen Jugendlichen unterschied der FBB-ANZ ebenso moderat zwischen Angstpatient_innen und Patient_innen mit externalisierenden Störungen (AUC = .76), jedoch nicht zwischen Angstpatient_innen und Patient_innen mit depressiven Episoden (AUC $=.65$; siehe Tabelle 3 ).

Patient_innen mit Trennungsangst oder sozialen Phobien zeigten jeweils höhere Subskalenmittelwerte als Patient_innen mit anderen Angststörungen. Patient_innen mit generalisierter Angststörung zeigten nur in der Gruppe der 12;00-17;00-Jährigen höhere Subskalenmittelwerte im Vergleich zu anderen Angststörungen. Patient_innen mit spezifischen Phobien unterschieden sich auf der Subskala Spezifische Phobie nur in der Gruppe der 4;00-11;11-Jährigen, jedoch nicht in der Gruppe der 12;00-17;11-Jährigen. Die Diskriminationsfähigkeit von Trennungsängsten und anderen Angststörungen in der Gruppe der 4;00-11;11-Jährigen $(\mathrm{AUC}=.84)$ und der 12;00-17;11-Jährigen $(\mathrm{AUC}=.87)$ fiel gut aus. Im Kindesalter zeigte sich eine geringere Diskriminationsgenauigkeit zwischen generalisierter Angststörung (AUC $=.67)$, sozialen Phobien (AUC $=.66$ ) und spezifischen Phobien (AUC $=.69$ ) und jeweils anderen Angststörungen. Im Jugendalter zeigte sich ebenso eine geringere Diskriminationsfähigkeit zwischen generalisierter Angststörung (AUC $=.61$ ), sozialen Phobien (AUC $=.70)$ und spezifischen Phobien (AUC $=.54$ ) und jeweils anderen Angststörungen.

Tabelle 3 beinhaltet die Sensitivitäten, Spezifitäten, positiven/negativen prädiktiven Werte und die dazugehörigen Cut-off-Werte, erstens bei einer festgelegten Sensitivität von mindesten $90 \%$ und zweitens bei einem optimalen Verhältnis von Sensitivität und Spezifität gemessen anhand des Youden-Index.

\section{Diskussion}

Ziel der vorliegenden Studie war die Überprüfung der Validität des Screeningergebnisses im FBB-ANZ zur Unterscheidung von Angststörungen und depressiven Episoden und externalisierenden Störungen sowie hinsichtlich differenzialdiagnostischer Überlegungen zur Abgrenzung einer spezifischen Angststörung bei Angstpatient_innen. Die psychometrische Re-Evaluation des FBB-ANZ bestätigt eine gute Konstruktion des Screeningfragebogens. Die Trennschärfen und internen Konsistenzen der Gesamtund Subskalen replizieren bisherige psychometrische Untersuchungsergebnisse (Döpfner et al., 2008; Dose, GörtzDorten, Breuer, Goletz \& Döpfner, 2015; Dose, Görtz-Dorten, Goletz \& Döpfner, 2018). Die Fit-Indizes der konfirmatorischen Faktorenanalyse zeigen eine gute bis akzeptable Struktur der Sub- und Gesamtskalen und bestätigen die bisherigen Ergebnisse zur Faktorenstruktur des Screeningfragebogens (Dose et al., 2018). Die mittleren, positiven Korrelationen der Gesamtskala Angst und Zwang des FBB-ANZ mit den CBCL-Skalen unterstützen die externe Validität des Fragebogens.

Die Validität des Screeningergebnisses im Rahmen der diagnostischen Mitbeurteilung von Angststörungen wurde durch ROC-Analysen untersucht. Die AUC-Werte deuten auf eine moderate Differenzierungsfähigkeit des FBB-ANZ zwischen Patient_innen mit Angststörungen und Patient innen mit externalisierenden Störungen in beiden Altersgruppen hin. Gleiches gilt für die Abgrenzung von Angststörungen und depressiven Störungen im Kindesalter, wohingegen die Differenzierungsfähigkeit im Jugendalter deutlich schlechter ausfiel. Vergleichbare Ergebnisse fanden sich auch bezüglich eines anderen Elternfragebogens, dem Screen for Child Anxiety Related Emotional Disorders (SCARED; Birmaher et al., 1997; Birmaher et al., 1999), für den mit dem Fragebogen für Angststörungen (FAS; bearbeitet durch Steinhausen, 2005) auch eine deutsche Version vorliegt. Hier zeigte sich bei 9- bis 18-jährigen Patient innen bei der Differenzierung von reinen Angststörungen und depressiven Störungen eine AUC von .58, und bei der Abgrenzung zu externalisierenden Störungen eine AUC von .78 (Birmaher et al., 1997). Monga et al. (2000) berichteten bei der Unterscheidung von Patient_innen mit und ohne Angststörungen anhand des SCARED eine AUC von .67. Im Vergleich mit dem SCARED/FAS zeigen die vorliegenden FBB-ANZ-Ergebnisse ähnliche Klassifikationsgenauigkeiten von Angst- und anderen Störungen. Eine mögliche Erklärung für die schlechtere Abgrenzung von Angststörungen und depressiven Episoden sind überlappende, ambigue Symptome der verwandten Störungsbilder. Beispielsweise ist sozialer Rückzug als Symptom mit Angststörungen und depressiven Episoden assoziiert (Rubin, Coplan \& Bowker, 2009). Die Ursache des sozialen Rückzugs, ob depressions- oder angstbedingt, ist für die korrekte Klassifikation der Erkrankung relevant, im Screeningurteil durch Eltern jedoch nur schwer beurteilbar. Es erscheint somit nachvollziehbar, dass sich Angststörungen im elterlichen Screeningurteil schlechter von depressiven Episoden als von externalisierenden Störungen abgrenzen lassen.

Die Ergebnisse zur Unterscheidung verschiedener Angststörungen anhand des FBB-ANZ sind heterogen. In beiden Altersgruppen konnte nur die emotionale Störung mit Trennungsangst gut von anderen Angststörungen abgegrenzt werden. Eine Erklärung dafür ist die verhaltensnahe und gut beobachtbare Symptomatik von Trennungsängsten. Beispielsweise reagieren trennungsängstliche Patient_innen in Trennungssituationen mit intensiver Angst, Wutausbrüchen, Schreien oder Apathie und bewältigen die (bevorstehende) Trennung von der Bezugsperson nicht altersgemäß (In-Albon \& Walter, 2002). Im Ge- 
Tabelle 2. (Sub-)Skalenmittelwerte nach Diagnosegruppen und Alter

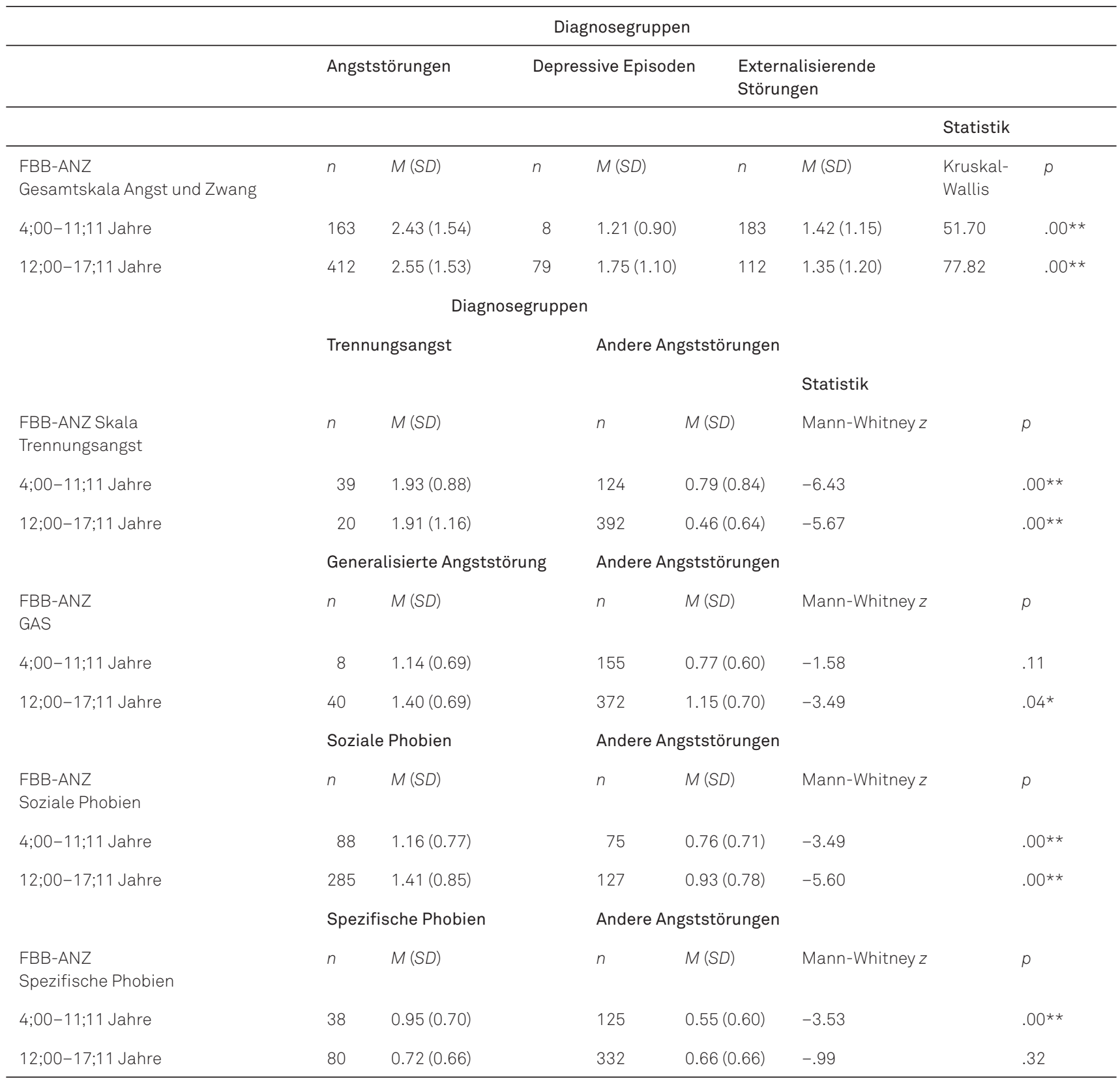

Anmerkungen: Stichprobengröße ( $n$ ) der Diagnosegruppen getrennt und Mittelwerte (M) und Standardabweichungen (SD) auf den der FBB-ANZ Gesamtskala und den Subskalen, getrennt nach Altersgruppen. Nonparametrische Teststatistik (bei > zwei Gruppen Kruskal-Wallis-Test; bei zwei Gruppen Mann-WhitneyU-Test); ${ }^{*} p<.05,{ }^{* *} p<.01$.

gensatz zu Symptomen der Trennungsangst ist bei anderen Angststörungen die Sichtbarkeit von Symptomen und somit die Beurteilung von außen eingeschränkt. Beispielsweise besteht bei der generalisierten Angststörung die Kernsymptomatik vor allem aus exzessiven Sorgen und Befürchtungen mit Katastrophisierungstendenzen (Lyneham, Rapee \& Schneider, 2004). Sorgen werden häufig nicht explizit verbalisiert und meist indirekt von beobachtbaren Symptomen wie Vermeidungs- und Rückversiche- rungsverhalten oder somatischen Beschwerden abgeleitet. Ebenso kann bei spezifischen Phobien die Sichtbarkeit von Symptomen eingeschränkt sein, wenn die angstauslösenden Situationen durch Patient innen vermieden werden können. Zusätzlich kann die gute Abgrenzung von Trennungsängsten bei den 4;00-11;11-Jährigen auch durch die hohe Prävalenz von Trennungsängsten im Kindesalter erklärt werden (Breton et al., 1999). Hohe Prävalenzen begünstigen die Wahrscheinlichkeit richtig-positi- 
Tabelle 3. Diskriminante Validität der Gesamtskala und der störungsspezifischen Unterskalen

\begin{tabular}{|c|c|c|c|c|c|c|c|c|}
\hline \multirow[b]{2}{*}{ 4;00-11;11 Jahre } & \multirow[b]{2}{*}{$n$} & \multirow[b]{2}{*}{$\mathrm{AUC}^{\mathrm{a}}[95 \% \mathrm{KI}]$} & \multicolumn{3}{|c|}{ Mindestens $90 \%$ Sensitivität } & \multicolumn{3}{|c|}{ Maximaler Youden-Index } \\
\hline & & & $\begin{array}{l}\text { Cut- } \\
\text { off }\end{array}$ & $\begin{array}{l}\text { Sensitivität/ } \\
\text { Spezifität }\end{array}$ & $\begin{array}{l}\mathrm{PPV}^{\mathrm{b}} / \\
\mathrm{NPV}\end{array}$ & $\begin{array}{l}\text { Cut- } \\
\text { off }\end{array}$ & $\begin{array}{l}\text { Sensitivität/ } \\
\text { Spezifität }\end{array}$ & $\begin{array}{l}\text { PPV/ } \\
\text { NPV }\end{array}$ \\
\hline $\begin{array}{l}\text { Angststörungen vs. depressive } \\
\text { Episoden }\end{array}$ & $163 / 8$ & $.77[.60-.93]$ & 0.75 & $.90 / .36$ & $.97 / .16$ & 0.95 & $.86 / .63$ & $.98 / .18$ \\
\hline $\begin{array}{l}\text { Angststörungen vs. } \\
\text { externalisierende Störungen }\end{array}$ & $163 / 183$ & $.72[.67-.77]$ & 0.75 & $.90 / .31$ & $.54 / .78$ & 1.25 & $.77 / .56$ & $.61 / .73$ \\
\hline $\begin{array}{l}\text { Trennungsangst vs. andere } \\
\text { Angststörungen }\end{array}$ & $39 / 124$ & $.84[.78-.90]$ & 0.56 & $.95 / .61$ & $.44 / .97$ & 0.79 & $.95 / .61$ & $.44 / .97$ \\
\hline $\begin{array}{l}\text { Generalisierte Angststörung vs. } \\
\text { andere Angststörungen }\end{array}$ & $8 / 155$ & $.67[.51-.83]$ & 0.22 & $1.00 / .22$ & $.06 / 1.00$ & 0.64 & $.88 / .48$ & $.08 / .99$ \\
\hline $\begin{array}{l}\text { Soziale Phobien vs. andere } \\
\text { Angststörungen }\end{array}$ & $88 / 75$ & $.69[.58-.74]$ & 0.15 & $.97 / .19$ & $.58 / 82$ & 0.36 & $.82 / .45$ & $.64 / .68$ \\
\hline $\begin{array}{l}\text { Spezifische Phobien vs. andere } \\
\text { Angststörungen }\end{array}$ & $38 / 125$ & $.69[.59-.78]$ & 0.14 & $.92 / .22$ & $.26 / .90$ & 0.64 & $.63 / .37$ & $.37 / .86$ \\
\hline 12;00-17;11 Jahre & & & & & & & & \\
\hline $\begin{array}{l}\text { Angststörungen vs. depressive } \\
\text { Episoden }\end{array}$ & $412 / 79$ & $.65[.59-.71]$ & 0.13 & $.91 / .22$ & $.86 / .32$ & 1.85 & $.62 / .65$ & $.90 / .25$ \\
\hline $\begin{array}{l}\text { Angststörungen vs. } \\
\text { externalisierende Störungen }\end{array}$ & $412 / 112$ & $.76[.71-.81]$ & 0.26 & $.91 / .35$ & $.84 / .51$ & 1.85 & $.62 / .78$ & $.91 / .36$ \\
\hline $\begin{array}{l}\text { Trennungsangst vs. andere } \\
\text { Angststörungen }\end{array}$ & 20/392 & $.87[.78-.95]$ & 0.58 & $.90 / .68$ & $.12 / .99$ & 1.64 & $.65 / .94$ & $.35 / .98$ \\
\hline $\begin{array}{l}\text { Generalisierte Angststörung vs. } \\
\text { andere Angststörungen }\end{array}$ & 40/372 & $.60[.51-.69]$ & 0.16 & $.96 / .19$ & $.11 / .99$ & 0.50 & $.98 / .19$ & $.11 / .99$ \\
\hline $\begin{array}{l}\text { Soziale Phobien vs. andere } \\
\text { Angststörungen }\end{array}$ & $285 / 127$ & $.67[.61-.73]$ & 0.11 & $.95 / .17$ & $.72 / .57$ & 1.07 & $.60 / .67$ & $.80 / .43$ \\
\hline $\begin{array}{l}\text { Spezifische Phobien vs. andere } \\
\text { Angststörungen }\end{array}$ & $80 / 332$ & $.54[.47-.60]$ & 0.05 & $.85 / .20$ & $.20 / 85$ & 0.21 & $.76 / .32$ & $.21 / .85$ \\
\hline
\end{tabular}

Anmerkungen: Ergebnisse der ROC-Analyse: AUC-Werte mit 95\%-Konfidenzintervall in Klammer. Optimale Cut-off-Werte, Sensitivitäten/Spezifitäten und PPV/NPN nach zwei Kriterien zur Unterscheidung der jeweils zu vergleichenden Patientengruppen getrennt nach Altersgruppen. Kriterium 1: Mindestanforderung an die Sensitivität $90 \%$. Kriterium 2: Optimales Verhältnis von Sensitivität und Spezifität.

a Area Under the Curve. ${ }^{b}$ Positiver prädiktiver Wert. ${ }^{c}$ Negativer prädiktiver Wert.

ver Screeningergebnisse. Bei 12;00-17;11-Jährigen zeigen sich jedoch vergleichbar gute Klassifikationsgenauigkeiten zum Kindesalter trotz niedrigerer Prävalenzen von Trennungsängsten im Jugendalter. Auch haben soziale Phobien und spezifische Phobien trotz hoher Prävalenzraten im Kindes- und Jugendalter im FBB-ANZ nicht zufriedenstellende Klassifikationsgenauigkeiten. In der Gesamtschau scheinen sich deshalb hohe Prävalenzraten nur bedingt als Erklärung der vorliegenden Ergebnisse bei Trennungsängsten zu eignen

Eine weitere Erklärung der heterogenen Klassifikationsgenauigkeit des FBB-ANZ hinsichtlich verschiedener Angststörungen liefert die Anzahl der Items und die Itemauswahl. Störungsübergreifende Screeningfragebögen wie der FBB-ANZ enthalten aus zeitökonomischen Gründen meist eine begrenzte Anzahl von Items, welche die wichtigsten Kriterien der Störungsbilder erfassen. Es erscheint jedoch begründet, dass eine größere Itemanzahl zu einer besseren inhaltlichen Abbildung der Subskalen führt und somit die Klassifikationsgenauigkeit erhöht. Beispielsweise hat die Skala Trennungsangst nicht nur die meisten Items, sondern weist auch die beste Klassifikationsgenauigkeit auf. Es erscheint möglich, dass durch die größere Itemanzahl die Skala Trennungsangst inhaltlich besser abgebildet wurde und somit die differenzialdiagnostische Abgrenzung von Trennungsängsten und anderen Angststörungen begünstigte. Der inhaltliche Fokus der Items im FBB-ANZ kann zudem die Abgrenzung von Angststörungen erschwert haben. Am Beispiel der generalisierten Angststörung zeigt sich, dass die Items der dazugehörigen Skala hauptsächlich 
die vegetativen Symptome des Krankheitsbilds abbilden. Es erscheint möglich, dass eine stärkere Berücksichtigung der Kernsymptomatik (z.B. exzessive Sorgen, Gefühl der Unkontrollierbarkeit der Sorgen, Rückversicherung, damit einhergehendes Vermeidungs- und Rückversicherungsverhalten) die Klassifikationsgenauigkeit der Skala zur Erfassung der generalisierten Angststörung in Abgrenzung zu anderen Angststörungen verbessern könnte.

Zuletzt verdeutlichen die vorliegenden Ergebnisse, dass beim Einsatz von Screeningfragebögen die zugrunde liegende klinische Fragestellung entscheidend für die Methodenwahl zur Bestimmung der optimalen Cut-off-Werte ist. Aus klinischer Perspektive dienen Screeningfragebögen der Abklärung von Verdachtsdiagnosen, sodass möglichst keine Hinweise auf eine Angststörung übersehen werden sollen. Hilfreich ist hier eine kriteriumsorientierte Testwertinterpretation (Moosbrugger \& Kelava, 2012), bei welcher Cut-off-Werte durch ein bestimmtes Kriterium, beispielsweise eine Mindestanforderung an die Sensitivitäten, festgelegt werden. Dies hat in der Regel niedrigere Cut-offWerte zur Folge. Nachteil niedrigerer Cut-off-Werte ist jedoch, dass bei leichter, subklinischer Symptomatik falschpositive Ergebnisse wahrscheinlich sind (siehe Bühner, 2011; Moosbrugger \& Kelava, 2012). Dieser Zusammenhang zeigt sich in den vorliegenden Daten bei der Wahl hoher Sensitivitäten von mindesten $90 \%$ als festes Kriterium. Bei allen untersuchten Angststörungen ist der positive prädiktive Wert niedrig, was auf eine hohe Rate falsch-positiver Ergebnisse bei der Wahl des entsprechenden Cut-OffWerts hinweist. Aus ökonomischer Perspektive erscheint deshalb eine stärkere Mitberücksichtigung hoher Spezifitäten sinnvoll, um überflüssige Diagnostik zu vermeiden. In den vorliegenden Daten zeigt sich jedoch, dass sich bei stärkerer Berücksichtigung der Spezifität, beispielsweise bei der Wahl des Cut-offs anhand der optimalen Berücksichtigung von Sensitivtät und Spezifität (maximaler Youden-Index; Moosbrugger \& Kelava, 2012), der positive prädiktive Wert nicht wesentlich verbessert, sodass die Reduktion falsch-positiver Ergebnisse nicht erreicht wird. In der Gesamtschau der Ergebnisse scheint der klinische Nutzen von Screeningfragebögen mit hohen Sensitivitäten bei der Abklärung von Verdachtsdiagnosen zu überwiegen, weshalb niedrige Cut-off-Werte gewählt werden sollten.

\section{Limitation}

Eine erste Einschränkung sind die abweichenden Fallzahlen der einzelnen Gruppen. Vor allem die Gruppe der 4;00-11;00-Jährigen mit depressiven Störungen ohne Angststörungen ist sehr klein, da depressive Erkrankungen bei Kindern seltener als im Jugendalter und meist ko- morbid mit Angststörungen auftreten (Angold, 1988; Cummings et al., 2014; Fleming \& Offord, 1990). Zusätzlich deutet das Konfidenzintervall des AUC-Werts auf eine große Fehlervarianz hin, was die statistische Aussagekraft der Ergebnisse in dieser Gruppe einschränkt.

Zweitens wurde innerhalb der Altersgruppen keine Aufteilung nach Geschlecht vorgenommen, da die jeweiligen Gruppen dann zu geringe Fallzahlen aufgewiesen hätten. Geschlechtseffekte können deshalb basierend auf dieser Studie nicht beurteilt werden.

Eine dritte Einschränkung ist die Einteilung in nur zwei Altersgruppen, was die Unterschiede zwischen den engen Entwicklungszeitfenstern im Kindes- und Jugendalter nicht differenziert genug abbilden könnte. Die Einteilung wurde aufgrund inhaltlicher und praktischer Erwägungen getroffen und die Grenze zwischen dem Kindes- und Jugendalter analog zur CBCL bei 12 Jahren festgelegt. Zur besseren Vergleichbarkeit der Ergebnisse sollten zukünftig, bei größeren Fallzahlen pro Gruppe, kleinere Altersbereiche gewählt werden. Eine weitere Besonderheit ergibt sich hinsichtlich der zugrunde liegenden Stichprobe. In die vorliegende Untersuchung wurden überwiegend teil- und vollstationäre Patient_innen mit Angststörungen sowie zusätzlichen komorbiden psychiatrischen Störungen eingeschlossen. Im Vergleich dazu lieferten Birmaher et al. (1997) und Monga et al. (2000) AUC-Vergleichswerte von Angstpatient_innen ohne komorbide Störungen. Da bei Angststörungen komorbide Erkrankungen, vor allem depressive Störungen, größere Beeinträchtigungen zur Folge haben (Wittchen, Beesdo, Bittner \& Goodwin, 2003), ist eine schwerere Ausprägung der Angstsymptomatik der hier eingeschlossenen Angstpatient_innen wahrscheinlich und schränkt die Vergleichbarkeit der Studienergebnisse ein.

Als letzter Punkt schränkt auch die Aktualität des FBBANZ die Aussagekraft der vorliegenden Ergebnisse ein. Mit dem Fremdbeurteilungsbogen für Angststörungen (FBB-ANG) des Diagnostik-Systems für Psychische Störungen nach ICD-10 und DSM-5 für Kinder und Jugendliche - III (DISYPS-III; Döpfner \& Görtz-Dorten, 2017) ist bereits eine überarbeitete Version des Screeningfragebogens verfügbar. Hierbei ist zu beachten, dass die Items der Skalen Trennungsangst und Generalisierte Angststörungen des FBB-ANG identisch mit dem hier verwendeten FBB-ANZ sind, was einen direkten Transfer der vorliegenden Ergebnisse auf die überarbeitete Publikation des Screeningfragebogens ermöglicht. Abänderungen wurden im Vergleich zum FBB-ANZ an den Skalen Soziale Phobien (Fokussierung auf Vermeidungsverhalten) und Spezifische Phobien (Spezifizierung von Ängsten) vorgenommen und die Skala Agoraphobie ergänzt. Demnach beinhaltet auch die Gesamtskala Angst im FBB-ANG des DISYPS-III zusätzliche Symptome wie Panikattacken, sowie Items, die auf Panik- 
störung oder Agoraphobie hinweisen. Die Verbesserung der Validität der Gesamtskala Angst des FBB-ANG durch Abänderung der Skalen sollte in zukünftigen ROC-Studien untersucht werden.

\section{Zusammenfassung}

Die vorliegende Studie untersuchte die Validität von Angststörungen im Screeningurteil der Eltern erhoben durch den FBB-ANZ mittels ROC-Analysen. In der zusammenfassenden Beurteilung zeigt sich eine moderate Diskriminationsfähigkeit des Screeningfragebogens zwischen Angststörungen und externalisierenden Störungen bei Kindern und Jugendlichen und depressiven Episoden im Kindesalter. Innerhalb der Gruppe der Angststörungen lässt sich nur die emotionale Störung mit Trennungsangst gut von anderen Angststörungen abgrenzen. Die Ergebnisse verdeutlichen die Notwendigkeit einer kritischen Betrachtung und Interpretation von Screeningergebnissen basierend auf dem Elternurteil bei Angststörungen.

\section{Originalitätserklärung}

Hiermit wird bestätigt, dass es sich bei dem vorliegenden Manuskript um einen Qriginalbeitrag handelt und geistiges Eigentum der genannten Autor_innen ist. Das Manuskript wurde bisher nicht als Ganzes oder in Teilen in anderen Zeitschriften publiziert, das Copyright liegt bei den Autor_innen. Es wird versichert, dass das Manuskript während des Begutachtungsverfahrens keiner anderen deutschsprachigen Zeitschrift angeboten wird.

\section{Elektronisches Supplement}

Das elektronische Supplement (ESM) ist mit der OnlineVersion dieses Artikels verfügbar unter https://doi.org/10.1024/10.1024/1422-4917/a000795

ESM 1. Konfirmatorische Faktorenanalyse: Anpassungsgüte und Fit-Indizes (Tabelle).

ESM 2. Itemanalytische Untersuchung (Tabelle).

\section{Literatur}

Achenbach, T.M. (1991). Manual for the Child Behavior Checklist/4-18 and 1991 Profile. Burlington: University of Vermont, Department of Psychiatry.
American Psychiatric Association. (1994). Diagnostic and Statistical Manual of Mental Disorders Fourth Edition (DSM-IV). Washington DC: American Psychiatric Association.

Angold, A. (1988). Childhood and adolescent depression: I. Epidemiological and aetiological aspects. British Journal of Psychiatry, 152, 601-617. https://doi.org/10.1192/bjp.152.5.601

Arbeitsgruppe Deutsche Child Behavior Checklist. (1998), Elternfragebogen über das Verhalten von Kindern und Jugendlichen; deutsche Bearbeitung der Child Behavior Checklist (CBCL/4-18). Einführung und Anleitung zur Handauswertung. 2. Auflage mit deutschen Normen, bearbeitet von M. Döpfner, J. Plück, S. Bölte, K. Lenz, P. Melchers \& K. Heim. Köln: Arbeitsgruppe Kinder-, Jugend- und Familiendiagnostik (KJFD).

Aster, M., Neubauer, A. \& Horn, R. (Hrsg.). (2006). Wechsler Intelligenztest für Erwachsene WIE. Deutschsprachige Bearbeitung und Adaptation des WAIS-III von David Wechsler (2. korr. Aufl.) Frankfurt a. M.: Pearson Assessment.

Barkmann, C. \& Schulte-Markwort, M. (2007). Psychische Störungen im Kindes- und Jugendalter. Epidemiologie und Diagnostik. Monatszeitschrift Kinderheilkunde, 155, 906-914.

Beesdo, K., Knappe, S. \& Pine, D. (2009). Anxiety and anxiety disorders in children and adolescents: Developmental issues and implications for DSM-V. Psychiatric Clinics, 32, 483-525. https://doi.org/10.1016/j.psc.2009.06.002

Beidel, D.C., Turner, S.M. \& Morris, T.L. (1999). Behavioral treatment of childhood social phobia. Journal of Consulting and Clinical Psychology, 68, 1072-1080. https://doi.org/10.1037/0022006X.68.6.1072

Beidel, D.C., Turner, S.M., Young, B.J., Ammerman, R.T., Sallee, F.R. \& Crosby, L. (2007). Psychopathology of adolescent social phobia. Journal of Psychopathology and Behavioral Assessment, 29, 46-53. https://doi.org/10.1007/s10862-006-902 1-1

Birmaher, B., Brent, D.A., Chiappetta, L., Bridges, J. Monga, S. \& Baugher, M. (1999). Psychometric properties of the Screen for Child Anxiety Related Emotional Disorders (SCARED): A replication study. Journal of the American Academy of Child \& Adolescent Psychiatry, 38, 1230-1236. https://doi.org/10.1097/0000 4583-199910000-00011

Birmaher, B., Khetarpal, S., Brent, D., Cully, M., Balach, L., Kaufman, J. et al. (1997). The Screen for Child Anxiety Related Emotional Disorders (SCARED): Scale constructions and psychometric characteristics. Journal of the American Academy of Child \& Adolescent Psychiatry, 36, 545-553. https://doi.org/10.1097/00 004583-199704000-00018

Bortz, J. \& Döring, N. (2006). Statistik für Human- und Sozialwissenschaftler (6. Aufl.). Berlin: Springer-Lehrbuch. https://doi. org/10.1007/978-3-642-12770-0

Breton, J.-J., Bergeron, L., Valla, J.-P., Berthiaume, C., Gaudet, N., Lambert, M. et al. (1999). Quebec child mental health survey: prevalence of DSM-III-R mental health disorder. Journal of Child Psychology and Psychiatry, and Allied Disciplines, 40, 375384. https://doi.org/10.1111/1469-7610.00455

Brown, M. \& Cudeck, R. (2016). Alternative ways of assessing model fit. Sociological Methods \& Research, 21, 230-258. https:// doi.org/10.1177\%2F0049124192021002005

Bühner, M. (2011). Einführung in die Test- und Fragebogenkonstruktion (3. akt. Aufl.). München: Pearson Studium.

Cummings, C.M., Caporino, N.E. \& Kendall, P.C. (2014). Comorbidity of anxiety and depression in children and adolescents: 20 years after. Psychological Bulletin, 140, 816-845. https://doi. apa.org/doi/10.1037/a0034733

Döpfner, M. \& Görtz-Dorten, A. (2017). Diagnostik-System für Psychische Störungen nach ICD-10 und DSM-5 für Kinder und Jugendliche - III (DISYPS-III). Bern: Hogrefe. 
Döpfner, M., Görtz-Dorten, A. \& Lehmkuhl, G. (2008). DiagnostikSystem für psychische Störungen nach ICD-10 und DSM-IV für Kinder und Jugendliche - II. Bern: Huber.

Dose, C., Görtz-Dorten, A., Breuer, D., Goletz, H. \& Döpfner, M. (2015). Die Erfassung von Ängsten von Kindern/Jugendlichen und ihren Eltern. Eine Analyse mit den Angstfragebögen aus dem Diagnostik-System (DISYPS-II). Diagnostica, 61, 197-209. https://doi.org/10.1026/0012-1924/a000126

Dose, C., Görtz-Dorten, A., Goletz, H. \& Döpfner, M. (2018). Anxiety in children and adolescents rated by patients, parents, and teachers: Factor structure and psychometric properties of an ICD-10 and DSM-IV-based rating scale in a large clinical sample. Journal of Child and Family Studies, 27, 3185-3199. https:// doi.org/10.1007/s10826-018-1160-y

Essau, C.A., Conradt, J. \& Petermann, F. (2000). Frequency, comorbidity, and psychosocial impairment of depressive disorders in adolescents. Journal of Adolescent Research, 15, 470-481. https://doi.org/10.1177/0743558400154003

Flemming, J.E. \& Offord, D.R. (1990). Epidemiology of childhood depressive disorders: A critical review. Journal of the American Academy of Child \& Adolescent Psychiatry, 29, 571-580. https:// doi.org/10.1097/00004583-199007000-00010

Flora, D. B. \& Curran, P.J. (2004). An empirical evaluation of alternative methods of estimation for confirmatory factor analysis with ordinal data. Psychological Methods, 9, 466-491. http:// dx.doi.org/10.1037/1082-989X.9.4.466

Grills, A. E. \& Ollendick, T.H. (2003). Multiple informant agreement and the anxiety disorders interview schedule for parents and children. Journal of the American Academy of Child \& Adolescent Psychiatry, 42, 30-40. https://doi.org/10.1097/00004583200301000-00008

Hooper, D., Coughlan, J. \& Mullen, M. (2008). Structural equation modelling: Guidelines for determining model fit. Electronic Journal of Business Research Methods, 6, 53-60.

Hu, L. \& Bentler, P. (1999). Cutoff criteria for fit indexes in covariance structure analysis: Conventional versus new alternatives. Structural Equation Modeling: A Multidisciplinary Journal, 6, 1-55. https://doi.org/10.108/10705519909540118

lancu, I., Bodner, E. \& Ben-Zion, I.Z. (2015). Self-esteem, dependency, self-efficacy and self-criticism in social anxiety disorder. Comprehensive Psychiatry, 58, 165-171. https://doi.org/10.101 6/j.comppsych.2014.11.018

Ihle, W. \& Esser, G. (2002). Epidemiologie psychischer Störungen im Kindes- und Jugendalter. Prävalenz, Verlauf, Komorbiditäten und Geschlechterunterschiede. Psychologische Rundschau, 53, 159-169. https://doi.org/10.1026//0033-3042.53.4.159

In-Albon, T. \& Walter, D. (2020). Trennungsängste und Schulvermeidung. In M. Döpfner, M. Hautzinger \& M. Linden (Hrsg.), Verhaltenstherapiemanual: Kinder und Jugendliche. Psychotherapie: Praxis (S. 313-316). Berlin: Springer. https://doi.org/10.1007/ 978-3-662-58980-9_62

Kashani, J.H. \& Orvaschel, H. (1990). A community study of anxiety in children and adolescents. American Journal of Psychiatry, 147, 313-318. https://doi.org/10.1176/ajp.147.3.313

Kendall, P.C., Compton, S.N., Walkup, J.T., Birmaher, B., Albano, A. M., Sherrill, J. et al. (2010). Clinical characteristics of anxiety disordered youth. Journal of Anxiety Disorders, 24, 360-365. https://doi.org/10.1016/j.janxdis.2010.01.009

Last, C.G., Strauss, C.C. \& Francis, G. (1987). Comorbidity among childhood anxiety disorders. Journal of Nervous and Mental Disease, 175, 726-730. https://doi.org/10.1097/00005053-19871 2000-00004

Leite, W. \& Beretvas, S. N. (2010). The performance of multiple imputation for Likert-type items with missing data. Journal of Modern Applied Statistical Methods, 9, 64-74. https://doi.org/10.22 237/jmasm/1272686820
Li, C.-H. (2016). Confirmatory factor analysis with ordinal data: Comparing robust maximum likelihood and diagonally weighted least squares. Behavior Research Methods, 48, 936-949. https://doi.org/10.3758/s13428-015-0619-7

Lyneham, H.J., Rapee, R. M. \& Schneider, S. (2004). Generalisierte Angststörung. In S. Schneider (Hrsg.), Angststörungen bei Kindern und Jugendlichen (S. 197-236). Berlin: Springer. https:// doi.org/10.1007/978-3-642-18499-4_9

Monga, S., Birmaher, B., Chiappetta, L., Brent, D., Kaufman, J., Bridge, J. et al. (2000). Screen for Child Anxiety-Related Emotional Disorders (SCARED): Convergent and divergent validity. Depression \& Anxiety, 12, 58-91. https://doi.org/10.1002/1520-63 94(2000)12:2<85::AID-DA4>3.0.CO;2-2

Moosbrugger, H. \& Kelava, A. (2012). Testtheorie und Fragebogenkonstruktion (2. Aufl.). Heidelberg: Springer.

Ollendick, T.H., King, N.J. \& Muris, P. (2002). Fears and phobias in children: Phenomenology, epidemiology, and aetiology. Child and Adolescent Mental Health, 7, 98-106. https://doi. org/10.1111/1475-3588.00019

Orth, U., Robins, R. W. \& Roberts, B. W. (2008). Low self-esteem prospectively predicts depression in adolescence and young adulthood. Journal of Personality and Social Psychology, 95, 695-708. https://doi.org/10.1037/0022-3514.95.3.695

Petermann, F. (Hrsg.). (2009). Wechsler Preschool and Primary Scale of Intelligence - Third Edition. Deutschsprachige Adaption nach D. Wechsler (WPPSI-III). Frankfurt a. M.: Pearson Assessment.

Petermann, F. (Hrsg.). (2012). Wechsler Adult Intelligence Scale Fourth Edition. Deutschsprachige Adaption der WAIS-IV von D. Wechsler. Frankfurt a. M.: Pearson Assessment.

Petermann, F. \& Petermann, U. (Hrsg.). (2011). Wechsler Intelligence Scale for Children - Forth Edition. Deutschsprachige Adaption nach D. Wechsler (WISC-IV). Frankfurt a. M.: Pearson.

Ravens-Sieberer, U., Wille, N., Erhardt, M., Bettge, S., Wittchen, H.U., Rothenberger, A. et al. (2008). Prevalence of mental health problems among children and adolescents in Germany: Results of the BELLA study within the National Health Interview and Examination Survey. European Child \& Adolescent Psychiatry, 17, 22-33. https://doi.org/10.1007/s00787-008-1003-2

Ricken, G., Fritz, A., Schuck, K.-D. \& Preuß, U. (Hrsg.). (2007). HAWIVA-III. Hannover-Wechsler-Intelligenztest für das Vorschulalter - III. Bern: Huber.

Rossel, Y. (2012). lavaan: An R package for structural equation modeling. Journal of Statistical Software, 48, 1-36. https://doi. org/10.18637/jss.v048.i02

Rubin, D. (1987). Multiple imputation for nonresponse in surveys. Hoboken, N.J.: Wiley Classic Library.

Rubin, K. H., Coplan, R.J. \& Bowker, J.V. (2009). Social withdrawal in childhood. Annual Review of Psychology, 60, 141-171. https:// doi.org/10.1146/annurev.psych.60.110707.163642

Sayal, K., Prasad, V., Daley, D., Ford, T. \& Coghill, D. (2018). ADHD in children and young people: Prevalence, care pathways, and service provision. Lancet Psychiatry, 5, 175-186. https://doi. org/10.1016/S2215-0366(17)30167-0

Schermelleh-Engel, K., Moosbrugger, M. \& Müller, H. (2003). Evaluating the fit of structural equation models: Test of significance and descriptive goodness-of-fit measures. Methods of Psychological Research, 8, 23-74.

Sowislo, J.F. \& Orth, U. (2013). Does low self-esteem predict depression and anxiety? A meta-analysis of longitudinal studies. Psychological Bulletin, 139, 213-240. https://doi.org/10.1037/ a0028931

Tewes, U., Rossmann, P. \& Schallberger, U. (Hrsg.). (1999). Hamburg-Wechsler-Intelligenztest für Kinder - Dritte Auflage (HAWIK-III). Übersetzung und Adaption der WISC-III Wechsler Intelligence Scale for Children - Third Edition von D. Wechsler. Bern: Huber. 
van Buuren, S. \& Groothuis-Oudshoorn, K. (2011). Mice: Multivariate imputation by chained equations in R. Journal of Statistical Software, 45, 1-67. https://doi.org/10.18637/jss.v045.i03

Verduin, T. \& Kendall, P.C. (2003). Differential occurrence of comorbidity within childhood anxiety disorders. Journal of Clinical Child and Adolescent Psychology, 32, 290-295. https://doi. org/10.1207/S15374424JCCP3202_15

Vink, G., Lazendic, G. \& van Buuren, S. (2015). Partitioned predictive mean matching as a multilevel imputation technique. Psychological Test and Assessment Modeling, 57, 577-594.

Waite, P. \& Creswell, C. (2014). Children and adolescents referred for treatment of anxiety disorders: Differences in clinical characteristics. Journal of Affective Disorders, 167, 326-332. https://doi.org/10.1016/j.jad.2014.06.028

Wechsler, D. (1997). Wechsler Adult Intelligence Scale Test - Third Edition (WAIS-III). San Antonio: The Psychological Cooporation.

Weiß, R.H. (2006). CFT 20-R mit WS/ZF-R. Grundintelligenztest Skala 2 - Revision (CFT 20-R) mit Wortschatztest und Zahlenfolgetest - Revision (WX/ZF-R) (1. Aufl.). Göttingen: Hogrefe.

Weiß, R.H. \& Osterland, J. (2012). CFT 1-R. Grundintelligenztest Skala 1 - Revision (1. Aufl.). Göttingen: Hogrefe.

Wittchen, H.-U., Beesdo, K., Bittner, A. \& Goodwin, R. D. (2003). Depressive episodes - evidence for a causal role of primary anxiety disorders? European Psychiatry, 18, 384-393. https://doi. org/10.1016/j.eurpsy.2003.10.001

Wittchen, H.-U., Stein, M. B. \& Kessler, R. C. (1999). Social fears and social phobia in a community sample of adolescents and young adults: prevalence, risk factors and co-morbidity. Psychological Medicine, 29, 309-323. https://doi.org/10.1017/s0033291798 008174

\section{Historie}

Manuskript eingereicht: 11.01.2021

Nach Revision angenommen: 14.02.2021

Onlineveröffentlichung: 09.04.2021

\section{Interessenskonflikt}

Die Autoren haben keinen Interessenskonflikt.

\section{Förderung}

Open-Access-Veröffentlichung ermöglicht durch die Universitätsbibliothek der Goethe-Universität Frankfurt am Main.

\section{Andreas M. Mühlherr M.Sc.}

Klinikum der Goethe-Universität

Klinik für Psychiatrie, Psychosomatik und Psychotherapie des Kindes- und Jugendalters

Deutschordenstr. 50

60528 Frankfurt am Main

Deutschland

andreasmichael.muehlherr@kgu.de 